\title{
Successful Treatment of Chronic Erythema Nodosum with Vitamin B12
}

\author{
Ilia Volkov, MD, Inna Rudoy, MD, and Yan Press, $M D$
}

\begin{abstract}
A 38-year-old woman presented with painful lesions on both shins that first appeared a few days earlier. Physical examination revealed multiple red tender nodules on both legs. The patient had been treated with nonsteroidal anti-inflammatory drugs for having a few months without any improvement. The patient was referred to a dermatologist and a rheumatologist, who confirmed our diagnosis of chronic erythema nodosum (EN). She returned to the clinic 3 months later complaining of having numbness in the soles of her feet for a few weeks. Her serum vitamin B12 level was $118 \mathrm{pg} / \mathrm{mL}$ (normal range 135 to 911). After 4 weeks of twice weekly injections of vitamin B12 at a dose of $1000 \mathrm{mcg}$, there was a clear alleviation of the numbness, and the EN completely resolved without evidence of recurrence on follow-up. Because it seems that vitamin B12 caused resolution of EN in this case, we recommend that physicians consider testing for vitamin B12 deficiency in patients with EN.(J Am Board Fam Pract 2005; 18:567-9.)
\end{abstract}

Erythema nodosum (EN) presents with tender, inflamed, nodular lesions, usually appearing on the anterior aspect of the legs, which may be associated with a variety of diseases or caused by medications. We present the case of a 38-year-old woman with EN and numbness of the soles of the feet. The patient's serum B12 level was below normal at 118 $\mathrm{pg} / \mathrm{mL}$ (normal range 135 to 911). Although there is no proved causal relationship between vitamin B12 deficiency and EN, the condition completely resolved following treatment with vitamin B12 injections.

We conducted a comprehensive MEDLINE search using combinations of the following keywords: erythema nodosum, vitamin B12, vitamin B12 deficiency, treatment of erythema nodosum, vitamin B12 and dermatological problems, cobalamin. We did not find any reference relating to an association between the 2 conditions.

Submitted 23 February 2005; revised 4 June 2005; accepted 14 June 2005.

From the Department of Family Medicine, Faculty of Health Sciences, Ben-Gurion University of the Negev, Beer-Sheva, Israel.

Conflict of interest: none declared.

Corresponding author: Ilia Volkov, MD, Department of Family Medicine, Faculty of Health Sciences, Ben-Gurion University of the Negev, PO Box 84105, Beer-Sheva 84514, Israel (e-mail: r0019@zahav.net.il).

\section{Case Description}

A 38-year-old usually healthy woman presented with painful lesions on both shins that first appeared a few days earlier. She did not take any medicine on a regular basis or before the development of the rash and had not suffered from a fever in the previous days. Her medical history was unremarkable.

Physical examination was normal other than the above-mentioned rash. Specifically, the temperature was normal, there was no enlargement of the lymph nodes, no enlargement of the liver or spleen, and no signs of arthritis. There were multiple erythematous, tender nodules $(2$ to $6 \mathrm{~cm}$ ) on both legs on the skin examination. At repeat examination a week later, the nodules had become darker, more tense, and hard. They were still extremely sensitive to touch.

The results of blood tests (chemistry, complete blood count, antinuclear antibody, rheumatoid factor, complement, thyroid-stimulating hormone, sedimentation rate, human immunodeficiency virus, antistreptolysin, anti-tissue transglutaminase antibodies) were within the normal range. A chest radiograph and an abdominal sonogram were normal. Treatment was begun with a nonsteroidal anti-inflammatory drug (NSAID). After a few months, the patient discontinued NSAID treatment on her own because she felt it was ineffective. The patient was referred to a dermatologist and a rheumatologist, and both 
confirmed the diagnosis of chronic EN. She did not come back for further treatment at that time. She returned to the clinic 3 months later with numbness in the soles of her feet that had developed a few weeks earlier. Physical examination revealed impaired vibration sensation on the soles of the feet and signs of EN on her thighs. The patient related that she had maintained a vegetarian diet for the past 20 years. The serum vitamin B12 level was below normal at 118 $\mathrm{pg} / \mathrm{mL}$ (normal range 135 to 911 ). Vitamin B12 levels were measured by a microparticle enzyme immunoassay (AxSYM; Abbott Laboratories). Treatment was started with intramuscular injections of vitamin B12 at a dose of $1000 \mathrm{mcg}$ twice a week. At 4 weeks follow-up, there was a definite alleviation of the numbness and a feeling of warmth in the feet, and the EN had completely resolved. The patient stated that the rash, which had been present for 6 months, totally disappeared 2 to 3 weeks after initiation of vitamin B12 therapy. The patient continued to receive monthly intra-muscular injections of vitamin B12 at a dose of $1000 \mathrm{mcg}$ without any evidence of recurrence of EN. In 6 months we decided to change therapy to sublingual vitamin B12 1000 mcg (Solgar) twice a week. Follow-up levels of vitamin B12 were not determined because the patient has been a vegetarian for many years and diagnosed as suffering from a peripheral neuropathy, and as such requires supplementation on a chronic basis. Because the patient continues to receive vitamin $\mathrm{B} 12$ on a regular basis there is no value in a repeat blood test in this case.

\section{Discussion}

EN, a cutaneous reaction consisting of inflamed, tender, nodular lesions, is the most common type of the panniculitides. It is usually located on the anterior aspect of the lower extremities. EN can be associated with a broad spectrum of conditions, most commonly infections, sarcoidosis, rheumatologic disorders, inflammatory bowel disease, autoimmune disorders, malignancy, pregnancy, and medications. Typically it is manifested by the sudden onset of symmetrical, tender, erythematous, warm nodules, and raised plaques, usually located on the shins, ankles, and knees. Often the lesions are distributed bilaterally. At first, the nodules show a bright red color but within a few days they become livid red or purplish, and finally they exhibit a yellow or greenish appearance taking on the look of a deep bruise.

In $60 \%$ of $\mathrm{EN}$ cases, no cause is found. Vascular deposition of immunoglobulin complexes and complement has been hypothesized as the cause of septal panniculitis, but evidence of this pathophysiological process remains elusive.

Treatment of EN should be directed at the associated underlying condition, if identified. Usually, nodules of EN regress spontaneously within a few weeks, and bed rest is often sufficient. Aspirin, NSAIDs (such as oxyphenbutazone, indomethacin, or naproxen), and potassium iodide may be helpful, as well as drugs that enhance analgesia and facilitate resolution. Systemic corticosteroids are rarely indicated in $\mathrm{EN}$, and an underlying infection should be ruled out before they are administered. ${ }^{1}$

In recent years, physicians have become increasingly aware of many medical conditions associated with vitamin B12 deficiency, including neurological problems of various degrees of severity, with and without the usual or expected hematologic pathol$\mathrm{ogy}^{2}$; developmental problems of infants whose mothers have severe vitamin B12 deficiency ${ }^{3}$; psychiatric problems; and mental disturbances. ${ }^{4}$ Possible effects of vitamin B12 deficiency on multiple sclerosis, ${ }^{5}$ homocysteine, ${ }^{6}$ cutaneous hyperpigmentation, ${ }^{7}$ and recurrent aphthous stomatitis ${ }^{8}$ are currently being studied.

We know that not only vegans or vegetarians suffer from vitamin B12 deficiency, but also persons with low meat intake. ${ }^{9}$ Because it seems that vitamin $\mathrm{B} 12$ caused resolution of $\mathrm{EN}$ in this case, we recommend that physicians consider testing for vitamin B12 deficiency in patients with EN.

\section{References}

1. Requena L, Requena C. Erythema nodosum. Dermatol Online J 2002;8:4.

2. Solomon LR. Cobalamin-responsive disorders in the ambulatory care setting: unreliability of cobalamin, methylmalonic acid, and homocysteine testing. Blood 2005;105:978-85.

3. Sklar R. Nutritional vitamin B12 deficiency in a breast-fed infant of a vegan-diet mother. Clinical Pediatrics (Phila) 1986;25:219-21.

4. Kumar S. Vitamin B12 deficiency presenting with an acute reversible extrapyramidal syndrome. Neurol India 2004;52:507-9.

5. Mastronardi FG, Min W, Wang H, et al. Attenua- 
tion of experimental autoimmune encephalomyelitis and nonimmune demyelination by IFN- $\beta$ plus vitamin B12: treatment to modify notch-1/sonic hedgehog balance. J Immunol 2004;172:6418-26.

6. Stanger O, Herrmann W, Pietrzik K, et al. DACHLIGA homocystein (German, Austrian and Swiss Homocysteine Society): consensus paper on the rational clinical use of homocysteine, folic acid and B-vitamins in cardiovascular and thrombotic diseases: guidelines and recommendations. Clin Chem Lab Med 2003;41:1392-403.
7. Mori K, Ando I, Kukita A. Generalized hyperpigmentation of the skin due to vitamin B12 deficiency. J Dermatol 2001;28:282-5.

8. Volkov I, Rudoy I, Abu-Rabia U, Masalha T, Masalha R. Recurrent aphthous stomatitis responsive to vitamin B12 treatment. Can Fam Physician 2005;51:844-5.

9. Masalha R, Rudoy I, Volkov I, Yusuf N, Wirguin I, Herishana YO. Symptomatic dietary vitamin B12 deficiency in a nonvegetarian population. Am J Med 2002;112:413-6. 